\title{
RESEARCH
}

Open Access

\section{Conditioned medium derived from FGF-2- modified GMSCs enhances migration and angiogenesis of human umbilical vein endothelial cells}

Shanshan Jin 1,2,3, Chengzhe Yang ${ }^{4}$, Jiahui Huang ${ }^{1,2,3}$, Lianlian Liu ${ }^{1,2,3}$, Yu Zhang ${ }^{1,2,3}$, Shutong Li Li,2,3, Liguo Zhang $^{1,2,3}$, Qinfeng Sun ${ }^{1,2,3^{*+}}$ and Pishan Yang ${ }^{1,2,3^{*}}$ (i)

\begin{abstract}
Background: Angiogenesis plays an important role in tissue repair and regeneration, and conditioned medium (CM) derived from mesenchymal stem cells (MSC-CM) possesses pro-angiogenesis. Nevertheless, the profile and concentration of growth factors in MSC-CM remain to be optimized. Fibroblast growth factor-2 (FGF-2) has been proven to be an effective angiogenic factor. Thus, the aim of this study was to verify whether FGF-2 gene overexpression optimized CM from human gingival mesenchymal stem cells (hGMSCs) and whether such optimized CM possessed more favorable pro-angiogenesis effect.

Methods: First, FGF-2 gene-modified hGMSCs were constructed using lentiviral transfection technology (LV-FGF-2 ${ }^{+}$hGMSCs) and the concentration of angiogenesis-related factors in LV-FGF-2+-hGMSC-CM was determined by ELISA. Then, human umbilical vein endothelial cells (HUVECs) were co-cultured for 3 days with LV-FGF-2+-hGMSC-CM, and the expression level of placenta growth factor (PLGF), stem cell factor (SCF), vascular endothelial growth factor receptor 2 (VEGFR2) in HUVECs were determined by qRT-PCR, western blot, and cellular immunofluorescence techniques. The migration assay using transwell and in vitro tube formation experiments on matrigel matrix was conducted to determine the chemotaxis and angiogenesis enhanced by LV-FGF-2 ${ }^{+}-\mathrm{hGMSC}-\mathrm{CM}$. Finally, NOD-SCID mice were injected with matrigel mixed LV-FGF-2+-hGMSC-CM, and the plug sections were analyzed by immunohistochemistry staining with anti-human CD31 antibody.

Results: LV-FGF-2+-hGMSC-CM contained significantly more FGF-2, vascular endothelial growth factor A (VEGF-A), and transforming growth factor $\beta$ (TGF- $\beta$ ) than hGMSC-CM. HUVECs pretreated with LV-FGF-2 ${ }^{+}$-hGMSC-CM expressed significantly more PLGF, SCF, and VEGFR2 at gene and protein level than hGMSC-CM pretreated HUVECs. Compared with hGMSC-CM, LV-FGF-2 ${ }^{+}$-hGMSC-CM presented significantly stronger chemotaxis to HUVECS and significantly strengthened HUVECs mediated in vitro tube formation ability. In vivo, LV-FGF-2 ${ }^{+}-h G M S C-C M$ also possessed stronger promoting angiogenesis ability than hGMSC-CM.
\end{abstract}

Conclusions: Overexpression of FGF-2 gene promotes hGMSCs paracrine of angiogenesis-related growth factors, thereby obtaining an optimized conditioned medium for angiogenesis promotion.

Keywords: Conditioned medium, FGF-2, Angiogenesis, hGMSCs, HUVECs, Overexpression

\footnotetext{
* Correspondence: sunqinfeng@sdu.edu.cn; yangps@sdu.edu.cn

${ }^{\dagger}$ Qinfeng Sun and Pishan Yang contributed equally to this work.

'Department of Periodontology, School and Hospital of Stomatology,

Shandong University, No.44-1 Wenhua Road West, Jinan 250012, Shandong,

China

Full list of author information is available at the end of the article
}

(c) The Author(s). 2020 Open Access This article is distributed under the terms of the Creative Commons Attribution 4.0 International License (http://creativecommons.org/licenses/by/4.0/), which permits unrestricted use, distribution, and reproduction in any medium, provided you give appropriate credit to the original author(s) and the source, provide a link to the Creative Commons license, and indicate if changes were made. The Creative Commons Public Domain Dedication waiver (http://creativecommons.org/publicdomain/zero/1.0/) applies to the data made available in this article, unless otherwise stated. 


\section{Background}

Periodontitis, bone fractures, malformations, and surgical removal of tumors can cause oral and maxillofacial bone defects and interfere with normal function and configuration. Regeneration of these damaged bone tissue has become an important clinical problem [1]. Currently, bone grafts, including autografts, allografts, xenografts, and synthetic grafts, are the primary treatment modalities [2,3]. However, these bone grafts are limited in clinical application for varied reasons. Constraints of autografts lie in availability, prolonged operation time and accompanied donor site morbidity, while allografts bring patients at risk for infections and immune rejection $[4,5]$. The main drawback of xenografts and synthetic grafts is that they do not elicit any osteoinductive or osteogenic potential on their own [6]. The new options are needed to improve bone regeneration outcomes.

Mesenchymal stem cells (MSCs) offer a potential strategy for tissue repair and wound healing. MSCs are multipotent somatic stem cells, which can be obtained from a number of adult tissues including the bone marrow, adipose tissue [7], periodontal ligament [8,9], dental pulp [10, 11], and gingivae [12, 13]. MSCs have been used to treat a wide range of diseases, including bone defect regeneration [14]. However, MSCs transplantation has some limitations impeding its clinical use $[15,16]$, such as the poor engraftment, a relatively short life span and potential tumorigenesis of transplanted MSCs.

Recently, it has been discovered that, in addition to their direct differentiation into tissue-related cell type, MSCs behave a potential paracrine effect, which includes anti-inflammatory and/or immunomodulation; antiapoptosis; angiogenesis, stimulating the growth and differentiation of surrounding cells [17]; and chemoattraction properties $[18,19]$. Moreover, some scholars even argued that instead of directly transforming into a tissue-related cell type, transplanted MSCs promote the proliferation and differentiation of autologous MSCs by regulating the microenvironment of the lesion site [20, 21]. Therefore, the regenerative potential of MSC therapies has been found-at least in part-to be mediated via such paracrine actions. Furthermore, transplantation of conditioned medium $(\mathrm{CM})$ containing paracrine factors such as the insulin-like growth factor (IGF-1) and vascular endothelial growth factor (VEGF) has been reported to enhance wound healing and bone regeneration in animal models [22-24].

Although MSC-CM therapy appears to be a highly promising alternative to MSC transplantation, a major constrain in its clinical application is that the concentrations of growth factors in $\mathrm{CM}$ are too low for therapeutic use [20]. For that, some scholars manage to optimize the profile and concentration of growth factors and cytokines in MSC-CM by preconditioning MSCs with inflammatory stimuli [21] or growth factors [22].

There is growing evidence that tissue repair and regeneration are closely related to vascularization. Vascular endothelial growth factor A (VEGF-A), one of the most important regulators of angiogenesis [23] plays a crucial role during endochondral ossification. In VEGFA conditional knockout (CKO) mice, the lack of VEGFA in chondrocytes leads to impaired embryonic bone development, reduced removal of terminally differentiated hypertrophic chondrocytes, and death of a large number of cells in the femur. This evidence demonstrates the important role of angiogenesis during skeletal development [24]. The combined use of stem cells and endothelial cells has a stronger effect on repairing cardiac damage than using stem cells or endothelial cells alone [25]. Recent studies have shown that the improved vascularization strategies in vitro can greatly enhance the regeneration of bone tissue in vivo [26]. These backgrounds suggest that pro-angiogenesis is an important strategy for promoting tissue regeneration.

Numerous studies have confirmed that FGF-2 produced by mesoderm and neuroectodermal cells acts as an effective angiogenic factor to promote microvascular formation [27]. The ERK1/2-MAP kinase signaling pathway controls the proliferation of various cell types. Studies have shown that FGF-2 can promote the phosphorylation of ERK1/2 and thereby promote endothelial cell proliferation [28]. FGF-2 may increase the secretion of VEGF by vascular smooth muscle endothelial cells and Muller cells through the activation of PI3K, which promotes angiogenesis [29]. Moreover, FGF-2 has been proven to stimulate bone marrow mesenchymal stem cell (BMMSC) proliferation and maintain its multipotent while being added to the culture medium [30]. Our previous studies have also found that preconditioning with FGF-2 could enhance sustained proliferation and osteogenic differentiation capacity of stem cells from periodontal ligaments (PLDSCs) [31,32]. This implies that FGF-2 possesses a potential effect on angiogenesis and osteogenesis, and preconditioning with FGF-2 is one of the effective approaches to optimize MSC-CM.

Given the potential effect of FGF-2 on angiogenesis, osteogenesis, anti-inflammatory, immunoregulatory, and osteogenic effect of MSC-derived from gingivae (GMSCs), it is speculated that conditioned medium from FGF-2 overexpressed GMSCs may possess better angiogenesis and osteogenesis ability. Thus, in this study, FGF-2 gene-modified GMSCs (FGF-2 ${ }^{+}$-GMSCs) were constructed, angiogenesis-associated cytokines in CM from FGF-2 ${ }^{+}$-GMSCs were detected, and we used lentivirus as a vector to increase the concentration of FGF-2 of the conditioned medium by overexpressing the FGF-2 gene and increasing the amount of FGF-2 
secreted by the gingival mesenchymal stem cells, and hence promote the role of blood vessel formation.

\section{Materials and methods}

\section{Human GMSCs culture and identification}

The program adhered to guidelines of patients' consent for participation and research was supported by the Ethics Committee of Stomatological Hospital, Shandong University (No. 201712). Healthy human gingival tissues were obtained from 6 patients in the Stomatological Hospital of Shandong University and cultured in Dulbecco's modified Eagle's medium (DMEM, Hyclone, Logan, UT, USA) which contained $5 \%$ antibiotics (100 U/ml penicillin $\mathrm{G}, 10 \mathrm{mg} / \mathrm{ml}$ streptomycin). After washed five times with phosphate-buffered solution (PBS, Hyclone, Logan, UT, USA) and cut into small pieces about $1 \mathrm{~mm}^{2}$ within $2 \mathrm{~h}$, the tissues were seeded at the bottom of $25 \mathrm{~cm}^{2}$ cell culture flask (Corning, NY, USA) and cultured in DMEM with 20\% fetal bovine serum (FBS, BioInd, Kibbutz, Israel) and 1\% antibiotics at $37^{\circ} \mathrm{C}$ in a $5 \% \mathrm{CO}^{2}$ incubator. The primary cells were purified by limiting-dilution to obtain stem cells. Then, the stem cells were cultured in a $10-\mathrm{cm}$ cell-cultured dish in DMEM containing 10\% FBS. Cells were passaged with $0.25 \%$ trypsin/EDTA solution (Hyclone, Logan, UT, USA) when reached $80-90 \%$ confluency, and cells were used in the following experiments at passage 3. To identify the characteristics of GMSCs, colony formation unit, cell surface antigens, and in vitro osteogenic and adipogenic assays were performed as previously reported [12].

\section{LV-FGF-2 ${ }^{+}$-hGMSC construction}

The lentivirus vector construction carrying the FGF-2 (pHBLV-CMV-MCS-3FLAG-EF1-ZsGreen-T2A-PUROFGF-2) or control vector (pHBLV-CMV-MCS-3FLAGEF1-ZsGreen-T2A-PURO) and lentiviral generation were performed by the Hanbio Biotechnology Co., Ltd. (Shanghai, China). To construct LV-FGF-2 ${ }^{+}$-hGMSCs, $2 \times 10^{5}$ hGMSCs suspended in DMEM were plated into a 6-well plate. After $24 \mathrm{~h}$, cells were then incubated with the corresponding lentiviral particles $(\mathrm{MOI}=40)$ in $1 \mathrm{ml}$ of the regular culture medium supplemented with $6 \mu \mathrm{g} /$ $\mathrm{ml}$ polybrene. Four hours later, another $1 \mathrm{ml}$ of the regular culture medium was added to each well. The cells were incubated at $37^{\circ} \mathrm{C}$ for $24 \mathrm{~h}$ before the medium was switched to a regular culture medium. After $48 \mathrm{~h}$, green fluorescence was captured by a fluorescence microscope (Olympus, Tokyo, Japan). Stably transduced cells were selected with $1 \mu \mathrm{g} / \mathrm{ml}$ puromycin (Sigma, Louis, MO) and maintained in culture medium containing $0.5 \mu \mathrm{g} / \mathrm{ml}$ puromycin.

\section{Preparation of $\mathrm{CM}$}

hGMSCs, LV-vector ${ }^{+}$-hGMSCs, and LV-FGF-2 ${ }^{+}$hGMSCs $\left(1 \times 10^{6}\right.$ cell/dish $)$ were seeded in $10-\mathrm{cm}$ cellcultured dishes separately and cultured in DMEM with $10 \%$ FBS. At $80 \%$ confluence, the cells were washed three times with PBS and the media were replaced with a 10 -ml serum-free DMEM. After 3 days, the CM was centrifuged at $1000 \mathrm{rpm}$ for $10 \mathrm{~min}$, filtered with $0.22 \mu \mathrm{m}$ filter, and then concentrated using a $10-\mathrm{kDa}$ ultrafiltration centrifuge tube (Millipore, USA) at $8000 \mathrm{~g}$ of centrifugal force at $4{ }^{\circ} \mathrm{C}$ for $1 \mathrm{~h}$. The conditioned mediums were concentrated approximately 50 times. The collected $\mathrm{CM}$ was stored in $\mathrm{a}-80^{\circ} \mathrm{C}$ refrigerator for subsequent experiments.

\section{ELISA}

The relative expression levels of VEGF-A, TGF- $\beta$, and FGF-2 in CM were analyzed by using ELISA kits (Dakewe, Beijing, China), and the absorbance values were detected at $450 \mathrm{~nm}$ wavelength.

\section{HUVECs culture and pretreatment}

Human umbilical vein endothelial cells (HUVECs; ScienCell, USA) were cultured in endothelial cell medium (ECM; ScienCell, USA) with 5\% fetal bovine serum (FBS; ScienCell, USA), 1\% endothelial cell growth supplement (ECGS; ScienCell, USA), and 1\% penicillin/streptomycin solution (P/S; ScienCell, USA) in $37^{\circ} \mathrm{C}$ incubator with $5 \% \mathrm{CO}^{2}$ and $95 \%$ air. HUVECs were passaged when the cells reached $80-90 \%$ confluency. Cells were used for subsequent experiments at passages 4-7. The HUVECs were preconditioned by LV-FGF- $2^{+}-\mathrm{hGMSC}-\mathrm{CM}$, hGMSC-CM, or LV-vector ${ }^{+}$-hGMSC-CM for 3 days, and ECM (ScienCell, USA) without CM was chosen as the negative control. Then, the cells were cultured by ECM with 5\% fetal bovine serum (FBS; ScienCell, USA), 1\% endothelial cell growth supplement (ECGS; ScienCell, USA), and $1 \%$ penicillin/streptomycin solution (P/S, ScienCell) for 7 to 10 days.

\section{Quantitative real-time polymerase chain reaction (qRT- PCR)}

To identify the transfection efficiency by lentivirus, human GMSCs $\left(2 \times 10^{6}\right.$ cell/well $)$ transfected by lentivirus carrying the FGF-2 gene or control vector were seeded in 6-well plates containing DMEM with 10\% FBS. When the cells were fused to $80-90 \%$, total RNA of hGMSCs were isolated by RNAiso Plus (Takara, Kusatsu, Japan), extracted with chloroform-isopropanol, and precipitated with ethanol. After being quantified, the complementary DNA (cDNA) was reverse transcribed by using PrimeScript $^{\text {ti }}$ RT reagent Kit and DNA Eraser (Takara). qRTPCR quantitative assay was performed with LightCycler 96 Real-Time PCR System (Roche, Basel, Switzerland) 
by using TB GREEN ${ }^{\mathrm{ma}}$ Premix Ex Taq ${ }^{\mathrm{Tm}}$ II (Takara). The primer sequences and housekeeping gene GAPDH were listed in Table 1.

In order to identify the expression of angiogenesisrelated genes, HUVECs pretreated with hGMSC-CM, LV-vector ${ }^{+}$-hGMSC-CM, or LV-FGF-2 ${ }^{+}$-hGMSC-CM were cultured for another 7 to 10 days. Then, total RNA of HUVECs were isolated by RNAiso Plus (Takara, Kusatsu, Japan), extracted with chloroform-isopropanol, and precipitated with ethanol. After being quantified, the complementary DNA (cDNA) was reverse transcribed using PrimeScript ${ }^{\mathrm{mm}}$ RT reagent Kit and DNA Eraser (Takara). qRT-PCR quantitative assay was performed with LightCycler 96 Real-Time PCR System (Roche, Basel, Switzerland) using TB GREEN ${ }^{\text {sw }}$ Premix Ex Taq ${ }^{\text {Tw }}$ II (Takara). The primer sequences and housekeeping gene GAPDH were listed in Table 1.

\section{Western blot analysis of angiogenesis-related proteins} The pretreated HUVECs were washed by pre-cooling PBS three times, and the proteins were extracted by RIPA lysis containing 1\% PMSF (Solarbio, Beijing, China). The concentration of proteins was measured according to the BCA protein assay kit (Solarbio, Beijing, China). The proteins were boiled for $5 \mathrm{~min}$ in SDSPAGE loading buffer, and $30 \mu \mathrm{g}$ of proteins was loaded to each lane and separated by $12 \%$ SDS-PAGE gels and transferred onto polyvinylidene fluoride (PVDF; Millipore, Billerica, MA, USA) membranes. The membranes were blocked by $5 \%$ non-fat milk for $1 \mathrm{~h}$ and then immersed in primary antibodies overnight at $4{ }^{\circ} \mathrm{C}$; the primary antibodies are as follows: rabbit anti-GAPDH (1:10000; Proteintech, Chicago, IN, USA), rabbit antiPLGF (1:1000; Abcam, Cambridge, UK), rabbit anti-SCF (1:10000; Abcam), and rabbit anti-VEGFR2 (1:1000; Cell Signaling Technology, USA). The membranes were incubated with horseradish peroxidase-conjugated secondary antibodies (1:10000; Proteintech) for $1 \mathrm{~h}$. Chemiluminescence reagents (Millipore) were used for the development of band visualization. ImageJ software was used to analyzed the protein expression levels.

\section{Immunocytochemistry}

HUVECs were pretreated with hGMSC-CM, LVvector $^{+}$-hGMSC-CM, or LV-FGF-2 ${ }^{+}$-hGMSC-CM for 3

Table 1 Primer sequences for qRT-PCR

\begin{tabular}{lll}
\hline Gene & Forward (5'-3') & Reverse (5'-3') \\
\hline GAPDH & GCACCGTCAAGGCTGAGAAC & TGGTGAAGACGCCAGTGGA \\
FGF-2 & GAGCGACCCTCACATCAA & CGTTCAGTGCCACATACC \\
PLGF & TTGTCTGCTGGGAACGGCTCGT & CCGGCACACAGTGCAGATTCT \\
SCF & GACCTTGTGGAGTGCGTGAA & CTGGGTTCTGGGCTCTTGAAT \\
VEGFR2 & CAAGTGGCTAAGGGCATGGA & ATTTCAAAGGGAGGCGAGCA \\
\hline
\end{tabular}

days. HUVECs were fixed with $4 \%$ paraformaldehyde (BioSharp) for $15 \mathrm{~min}$, then permeabilized the cells by 0.2\% Triton-X-100 (Dingguo, Beijing, China) for $15 \mathrm{~min}$. After being blocked with $10 \%$ goat serum for $1 \mathrm{~h}$, cells were incubated with rabbit anti-VEGFR2 primary antibody (1:400; Cell Signaling) overnight. After washed three times with PBS, cells were incubated with goat anti-rabbit IgG secondary antibody (1:500; Proteintech) for $1 \mathrm{~h}$ at $37^{\circ} \mathrm{C}$. 2-(4-amidinophenyl)-6-indolecarba midine dihydrochloride (DAPI; Proteintech) was used to stain nuclei. Images were captured with a fluorescence microscope (OLYMPUS IX73, Tokyo, Japan) in the darkroom.

\section{Cell migration assay}

To detect the effect of LV-FGF-2 ${ }^{+}$-hGMSC-CM on migration ability of HUVECs, $8 \times 10^{4}$ HUVECs were seeded at the upper chamber of the transwell chambers with 8$\mu \mathrm{m}$ pores (Corning, NY, USA) containing $200 \mu \mathrm{l} \mathrm{ECM}$ with $0.1 \% \mathrm{FBS}$, and the lower chamber was injected with $500 \mu \mathrm{l}$ ECM containing 0.1\% FBS, 0.1\% FBS $+10 \mu \mathrm{l}$

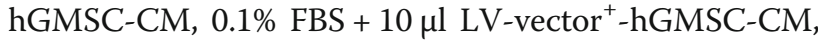
$0.1 \% \mathrm{FBS}+10 \mu \mathrm{l} \mathrm{LV-FGF-2}{ }^{+}$-hGMSC-CM, or $5 \% \mathrm{FBS}$, respectively. After incubating at $37^{\circ} \mathrm{C}$ for $20 \mathrm{~h}$, the HUVECs on the upper surface of the upper chamber were cleaned with cotton swabs and the HUVECs on the lower surface of the upper chamber were fixed with $4 \%$ paraformaldehyde (Biosharp, China) for $15 \mathrm{~min}$ and then stained with $0.1 \%$ crystal violet solution (Sigma-Aldrich) for $10 \mathrm{~min}$. The stained cells were observed under an inverted microscope (Olympus, Tokyo, Japan) at $\times 400$ magnification.

\section{Tube formation assay}

To visually analyze the angiogenic ability of LV-FGF- $2^{+}-$ hGMSC-CM, a 60- $\mu \mathrm{l}$ matrigel matrix (Corning) was transferred to a 96-well plate and then incubated in $37^{\circ} \mathrm{C}$ incubator for $30 \mathrm{~min}$. The HUVECs $\left(3 \times 10^{4}\right.$ cells/ well) were seeded on the matrigel matrix with $400 \mu \mathrm{l}$ ECM containing 5\% FBS, 5\% FBS + $8 \mu \mathrm{l} \mathrm{hGMSC-CM,} \mathrm{5 \%}$ $\mathrm{FBS}+8 \mu \mathrm{l} \quad \mathrm{LV}$-vector ${ }^{+}$-hGMSC-CM, or $5 \% \mathrm{FBS}+8 \mu \mathrm{l}$ LV-FGF- $2^{+}$-hGMSC-CM, respectively, and incubated at $37^{\circ} \mathrm{C}$ for 4 to $24 \mathrm{~h}$. The tube-like structures were photographed under an inverted microscope (Olympus, Tokyo, Japan) at $\times 40$ magnification, and the total tube length and total branching length were analyzed by Image J software.

\section{Animal experiment and immunohistochemistry staining} NOD-SCID mice (6 to 8 weeks of age) from Beijing Vital River Laboratory Animal Technology Co., Ltd. (Beijing, China) were used to detect the ability of LV-FGF-2 ${ }^{+}$hGMSC-CM in promoting HUVEC angiogenesis in vivo. These NOD-SCID mice were assigned to four groups: 
matrigel group (500 $\mu \mathrm{l}$ matrigel), HUVEC group (500 $\mu \mathrm{l}$ matrigel $+5 \times 10^{6}$ HUVECs), hGMSC-CM group $(500 \mu \mathrm{l}$ matrigel $+5 \times 10^{6}$ HUVECs $+100 \mu \mathrm{l}$ hGMSCs-CM), and LV-FGF-2 ${ }^{+}$-hGMSC-CM group $(500 \mu \mathrm{l}$ matrigel + $5 \times 10^{6}$ HUVECs $+100 \mu$ l LV-FGF-2 ${ }^{+}$-hGMSC-CM). Matrigel or matrigel mixture was injected subcutaneously into the lower dorsal region of SCID mice. After 7 days, the matrigel plugs were removed, fixed in $4 \%$ paraformaldehyde for $24 \mathrm{~h}$, rinsed in running water for $4 \mathrm{~h}$, dehydrated, embedded in paraffin blocks, and sectioned into $5 \mathrm{~mm}$. Then, immunohistochemistry staining was performed according to the protocol of the immunohistochemical stain kit (ZCGB-BIO, Beijing, China). Briefly, after dewaxing and dehydration, the sections were immersed in primary anti-human CD31 antibody (1: 200; Bioss, Beijing, China) overnight and immersed in biotin secondary antibody for $30 \mathrm{~min}$. After staining with DAB stain solution (ZCGB-BIO, Beijing, China), the sections were counter-stained by using hematoxylin for $3 \mathrm{~min}$ and eosin for $20 \mathrm{~s}$. Images were captured under an inverted microscope (Olympus, Tokyo, Japan) at $\times 200$ and $\times 400$ magnification.

\section{Statistical analysis}

SPSS 19.0 was used to conduct all the statistical analyses. Data were expressed as mean \pm standard error of the mean (S. E. M) of three independent experiments. Differences among groups were evaluated by one-way ANOVA followed by Tukey HSD comparison test. $P$ values less than 0.05 were considered to be statistically significant.

\section{Results}

hGMSCs culture and identification

The hGMSCs were obtained by tissue block digestionlimited dilution method (Fig. 1a, b). To identify hGMSCs,

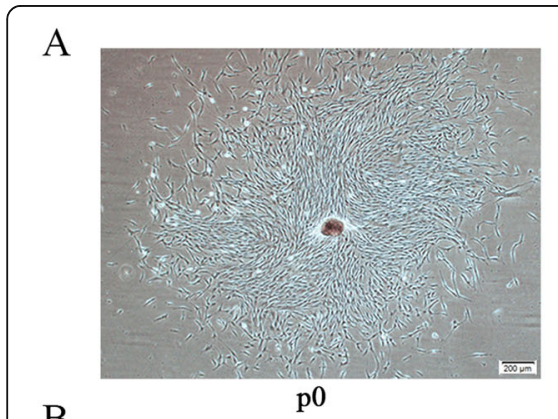

B

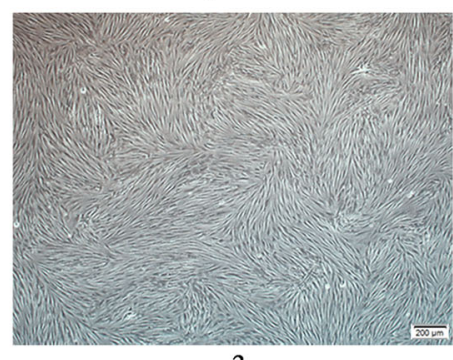

p3

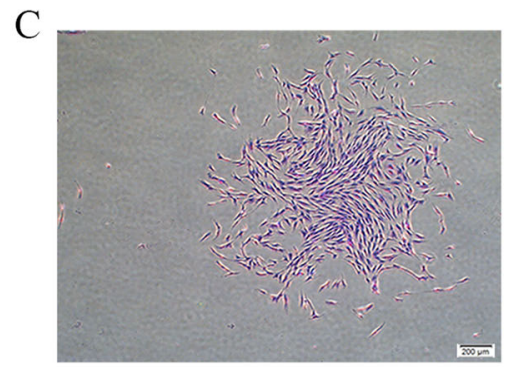

$\mathrm{D}$

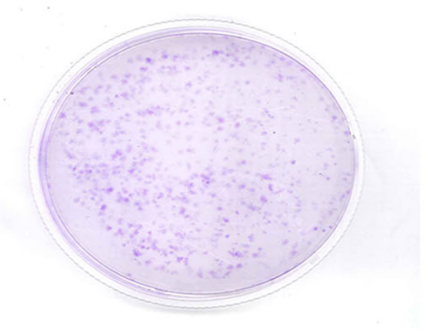

monoclone
E

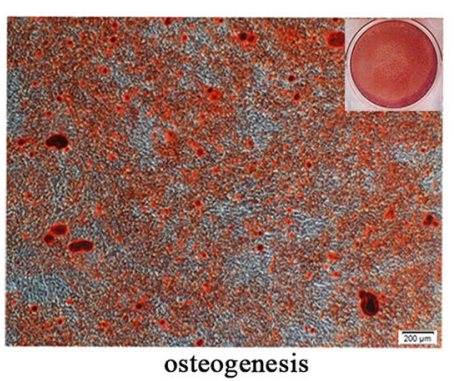

F

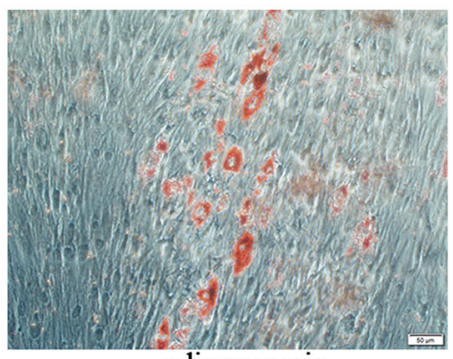

G
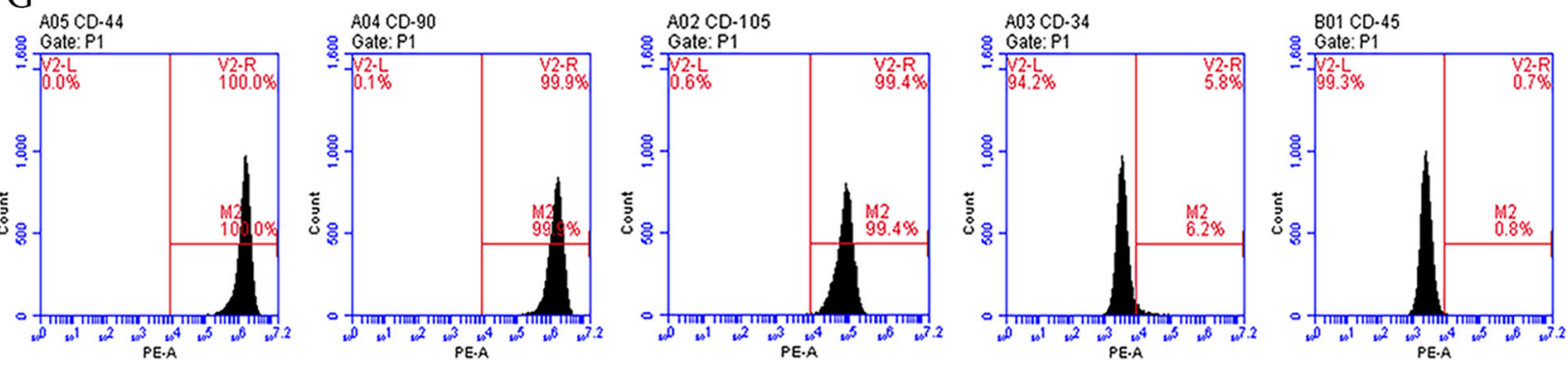

Fig. 1 Human GMSCS culture and identification. Human GMSCS were obtained by tissue block digestion-limited dilution method, and the thirdgeneration hGMSCs were used for subsequent experiments $(\mathbf{a}, \mathbf{b})$. In the flow cytometric analysis, hGMSCs positively expressed mesenchymal stem cell surface markers CD44 (100\%), CD90 (99.9\%), and CD105 (99.4\%), but negatively expressed hematopoietic stem cell surface markers CD34 $(5.8 \%)$ and CD45 (0.7\%). Negative control single peak is located on the left side of the vertical line (g). The clones were obtained by hGMSCs culturing for 12 days at 500 cells per dish (c, d). After 21 days of osteogenic induction, hGMSCs formed mineralized nodules that stained with alizarin red (e). After 21 days of adipogenic induction, hGMSCs formed lipid droplets stained by oil red O (f) 
the cell surface antigens were analyzed by flow cytometry; the positive indicators were CD44 (100\%), CD90 (99.9\%), and CD105 (99.4\%), and the negative indicators were CD34 (5.8\%) and CD45 (0.7\%) (Fig. 1g). hGMSCs have the potential of osteogenic differentiation and adipogenic differentiation (Fig. 1e, f). The colony-forming unit assay demonstrated that the human gingival mesenchymal stem cells have the ability to form clones (Fig. 1c, d).

\section{LV-FGF-2 transfection promotes FGF-2 gene expression} and FGF-2, VEGF-A, and TGF- $\beta$ paracrine of hGMSCs

The lentivirus vector $\mathrm{pHBLV}-\mathrm{CMV}-\mathrm{MCS}$-3FLAG-EF1ZsGreen-T2A-PURO-FGF-2 was successfully constructed as shown by the map of the plasmid (Additional file 1). The green fluorescence staining showed that the lentivirus was high in the hGMSCs when $\mathrm{MOI}=40$ (Fig. 2a). The qRT-PCR indicated that LVFGF-2 ${ }^{+}$-hGMSCs expressed higher FGF-2 compared with the LV-vector ${ }^{+}$-hGMSCs (Fig. 2b). ELISA assay showed that the concentrations of FGF-2 (Fig. 2c), VEGF-A (Fig. 2d), and TGF- $\beta$ (Fig. 2e) in LV-FGF$2^{+}$-hGMSC group significantly increased compared to those in $\mathrm{LV}_{\text {-vector }}^{+}$-hGMSC group and hGMSC group.
LV-FGF-2 ${ }^{+}$-hGMSC-CM preconditioning enhances the expression of angiogenesis-related factors in HUVECs SCF, PLGF, and VEGFR2 play significant roles in angiogenesis; thus, their expression in HUVECs undergoing different preconditioning treatments was assayed to reflect the angiogenesis potential of LV-FGF-2 ${ }^{+}$-hGMSCCM. As shown in Fig. 3, PLGF (Fig. 3a, d, g), SCF (Fig. 3b, e, h), and VEGFR2 (Fig. 3c, f, i) mRNA and protein expressions in HUVECs preconditioned by LV-FGF$2^{+}$-hGMSC-CM were significantly higher (except for VEGFR2 protein expression at 7th day) than in those preconditioned by LV-vector ${ }^{+}$-hGMSC-CM, hGMSC$\mathrm{CM}$, or negative control group. And PLGF, SCF, or VEGFR2 mRNA and protein expressions in the LVvector $^{+}$-hGMSC-CM group and hGMSC-CM group were also significantly higher than those in the negative control group (Fig. 3a-i).

Immunofluorescence staining presented a similar result. As shown in Fig. 3j and Fig. 3k, the VEGFR2 expression in HUVECs pretreated by LV-FGF-2 ${ }^{+}$-hGMSC$\mathrm{CM}$ was significantly higher than in those pretreated by hGMSC-CM and LV-vector ${ }^{+}$-hGMSC-CM. LV-vector ${ }^{+}$hGMSC-CM group and hGMSC-CM group also had a tendency to increase VEGFR2 expression, but no significant difference was arrived.
A

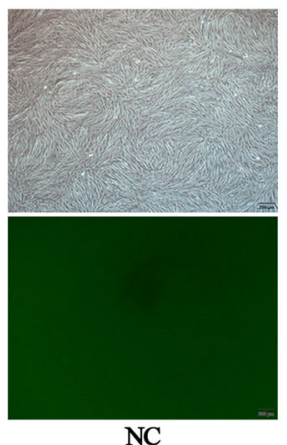

C

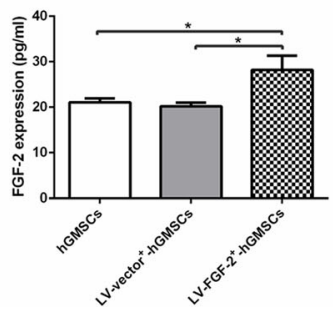

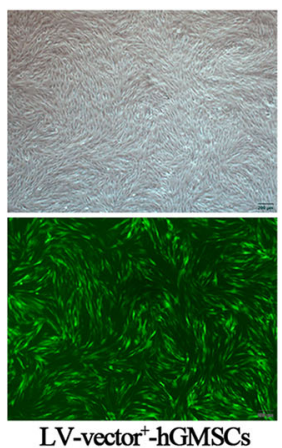

LV-vectort-hGMSCs

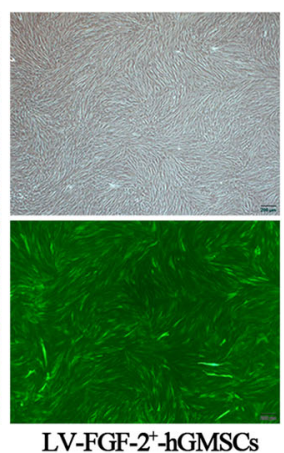

D

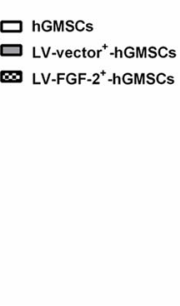

B
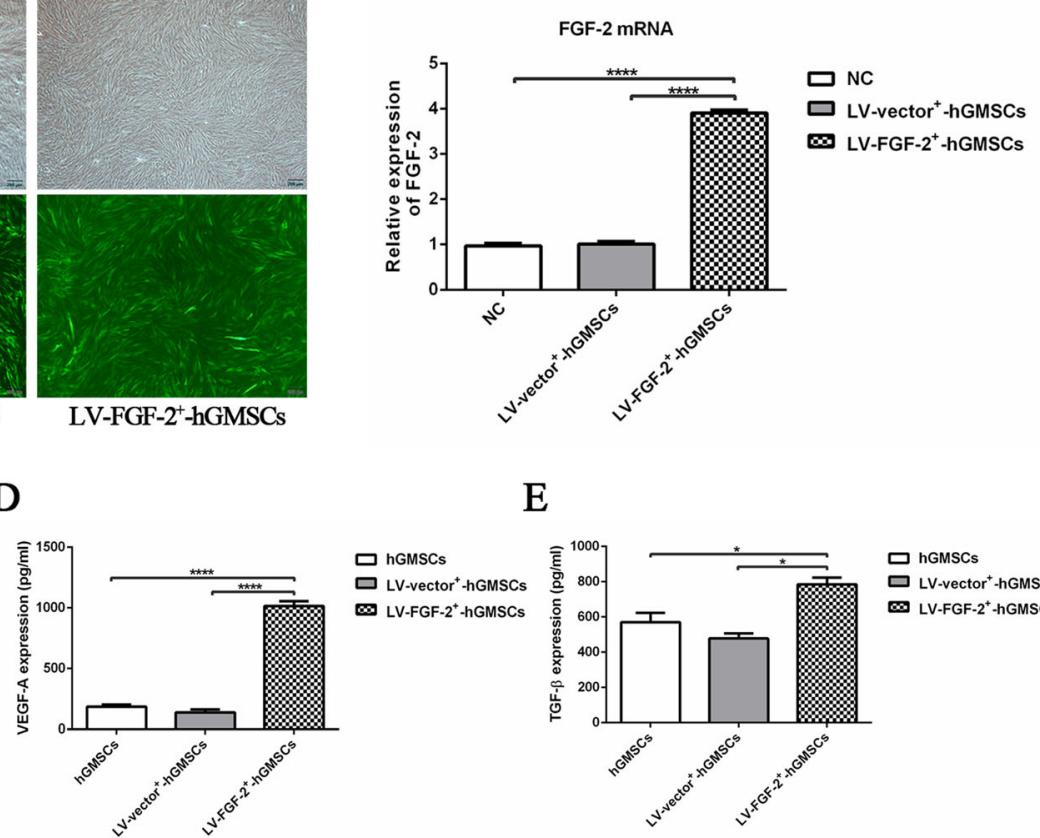

E

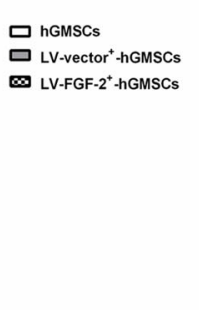

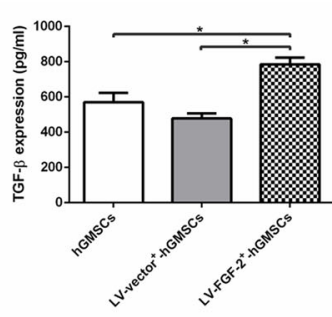

$\square$ hGMSCs

$\square$ LV-vector $^{+}$-hGMSCs $\infty$ LV-FGF-2+ $2^{+}$hGMSCs

Fig. 2 The lentiviral transfection efficiency and its effect on the paracrine of hGMSCs. Human GMSCs transfected with LV-vector ${ }^{+}$or LV-FGF-2 $(\mathrm{MOI}=40)$ were observed under an inverted microscope (a). The lentiviral transfection efficiency was detected by qRT-PCR (b). The transfected cells were cultured by free-serum medium for 3 days when they converged to $80-90 \%$. Angiogenesis-related factors FGF-2, VEGF-A, and TGF- $\beta$ in the supernatant were detected by ELISA. The concentration of FGF-2, VEGF-A, and TGF- $\beta$ in LV-FGF-2 ${ }^{+}-\mathrm{hGMSC-CM}$ is higher than other two groups; however, there was no statistical difference between hGMSC-CM and LV-vector ${ }^{+}-\mathrm{hGMSC}-\mathrm{CM}(\mathbf{c}-\mathbf{e}) .{ }^{*} p<0.05,{ }^{* * *} p<0.0001$ 





(See figure on previous page.)

Fig. 3 The effect of LV-FGF-2+-hGMSC-CM on the expression of angiogenesis-related factors in HUVECs. HUVECs were pretreated with hGMSCCM, LV-vector ${ }^{+}$-hGMSC-CM, or LV-FGF-2+-hGMSC-CM for 3 days (negative control group was cultured by ECM) and then re-cultured by ECM for 7 to 10 days. The mRNA and protein expressions of PLGF, SCF, and VEGFR2 were determined by qRT-PCR (a-c) and western blot (d-i). The VEGFR2 protein expression was also detected by immunofluorescence staining (VEGFR2, red fluorescent signals; DAPI, blue signals; $\times 400$ magnification) $\mathbf{j}$, k). ns = no significant difference, ${ }^{*} p<0.05,{ }^{* *} p<0.01,{ }^{* *} p<0.001,{ }^{* * *} p<0.0001$

\section{LV-FGF-2 ${ }^{+}$-hGMSC-CM promotes migration of HUVECs}

The migration assay by using transwell chambers revealed that $\mathrm{LV}-\mathrm{FGF}-2^{+}$-hGMSC-CM significantly increased the number of migrating HUVECs compared with ordinary medium, hGMSC-CM, or LVvector $^{+}$-hGMSC-CM. And the numbers of migrating HUVECs in the hGMSC-CM group and LV-vector ${ }^{+}$hGMSC-CM group were significantly more than those in the negative control group. As expected, the positive control group had the strongest promoting effect (Fig. $4 a-f)$.

\section{LV-FGF-2 ${ }^{+}$-hGMSC-CM increases tube formation}

To investigate the pro-angiogenic effects of LV-FGF$2^{+}$-hGMSC-CM, a tube formation assay was performed to observe vascular network formation by HUVECs. The results showed that at $4 \mathrm{~h}$, there was no significant difference in total tube length among hGMSC-CM group, LV-vector ${ }^{+}$hGMSC-CM, and LVFGF- $2^{+}$-hGMSC-CM group; however, the total tube length in these three groups was significantly longer than that in the negative control group (Fig. 5a, b). The total branching length in the LV-FGF-2 ${ }^{+}$hGMSC-CM group was significantly longer than that in the hGMSC-CM group and LV-vector ${ }^{+}$-hGMSC$\mathrm{CM}$ group, which was significantly longer than that in the negative control group (Fig. 5a, c). At $24 \mathrm{~h}$, both total tube length (Fig. 5a, d) and total branching length (Fig. 5a, e) in the LV-FGF-2 ${ }^{+}$-hGMSC-CM group were significantly longer than those in the LVvector $^{+}$-hGMSC-CM group and hGMSC-CM group, which were significantly longer than in the negative control group.

\section{LV-FGF- ${ }^{+}$-hGMSC-CM promotes angiogenesis in vivo}

To study the pro-angiogenesis ability of LV-FGF- $2^{+}$hGMSCs-CM in vivo, matrigel mixed with HUVECs, HUVECs with hGMSC-CM, or HUVECs with LVFGF-2 ${ }^{+}$-hGMSC-CM was injected subcutaneously into NOD-SCID mice and only matrigel was used as the control. After 7 days, blood vessels were analyzed by quantification of CD31 staining. As shown in Fig. 6,
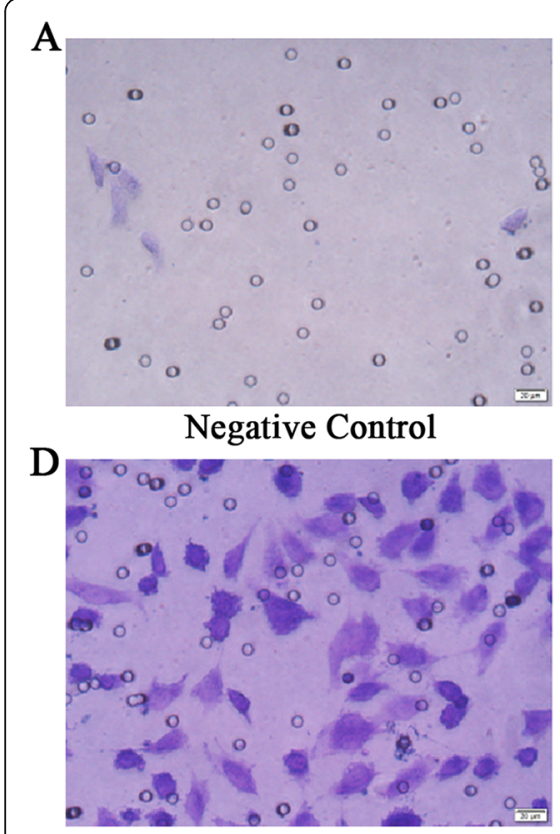

LV-FGF-2+hGMSC-CM

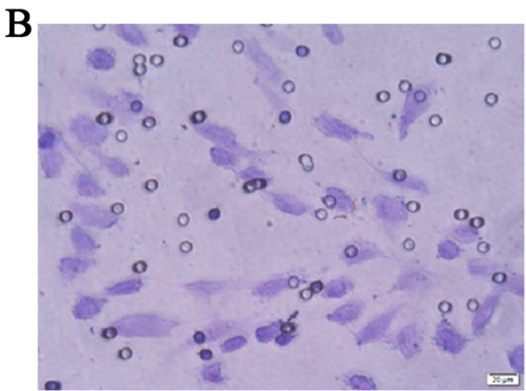

hGMSC-CM

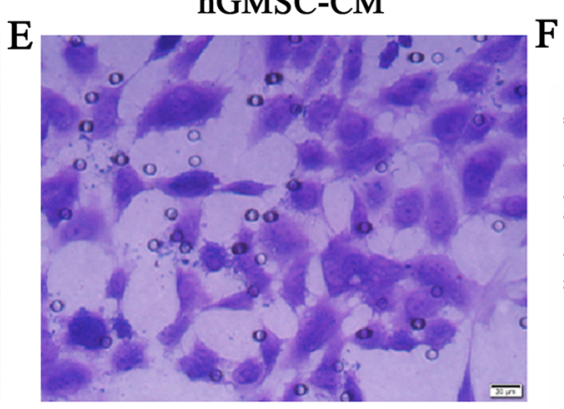

Positive Control

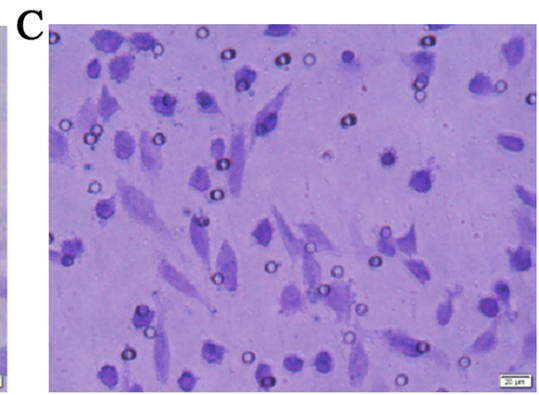

LV-vector ${ }^{+}$-hGMSC-CM

Fig. 4 The effect of LV-FGF-2+-hGMSC-CM on the migration of HUVECS. HUVECs were cultured in the upper chamber of the transwell chambers with $0.1 \% \mathrm{FBS}$, and $0.1 \% \mathrm{FBS}(\mathrm{NC}), 0.1 \% \mathrm{FBS}+$ hGMSC-CM, 0.1\% FBS + LV-vector ${ }^{+}$-hGMSC-CM, 0.1\% FBS + LV-FGF-2+-hGMSC-CM, or 5\% FBS (PC) was injected in the lower chamber. After $20 \mathrm{~h}$, the number of cells on the lower surface of the upper chamber was counted under an inverted microscope at $\times 400$ magnification (a-e) and statistically analyzed among five groups $(\mathbf{f}) .{ }^{* *} p<0.01,{ }^{* * *} p<0.001,{ }^{* * * *} p<0.0001$ 


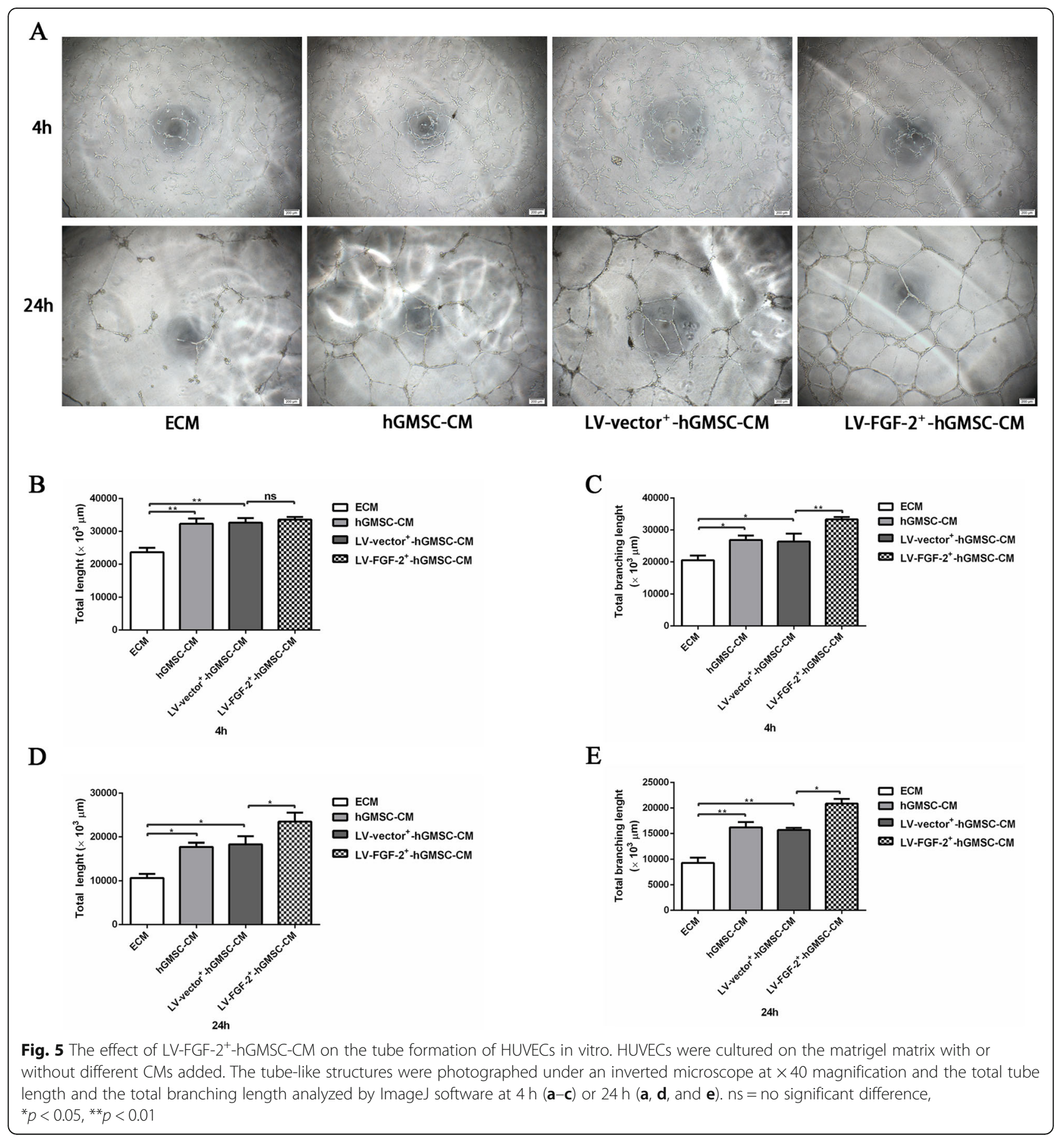

compared with plug enriched hGMSC-CM, HUVECs, or plug control, the plug-enriched LV-FGF-2 ${ }^{+}$hGMSC-CM showed a higher anti-CD31-positive capillary density (Fig. 6a, b). Although the anti-CD31positive capillary density in plug-enriched hGMSCs was higher than that in the plug with HUVECs, there was no statistical difference between them (Fig. 6a, b). In summary, the conditioned medium derived from FGF-2 gene overexpressed hGMSCs had a stronger ability promoting angiogenesis than that derived from hGMSCs.

\section{Discussion}

In this study, in order to optimize CM for the enhanced potential of angiogenesis, we used lentivirus as a vector to overexpress the FGF-2 gene into hGMSCs. Results demonstrated that FGF-2 gene transfection increased the expression and secretion of angiogenesis-related factors 


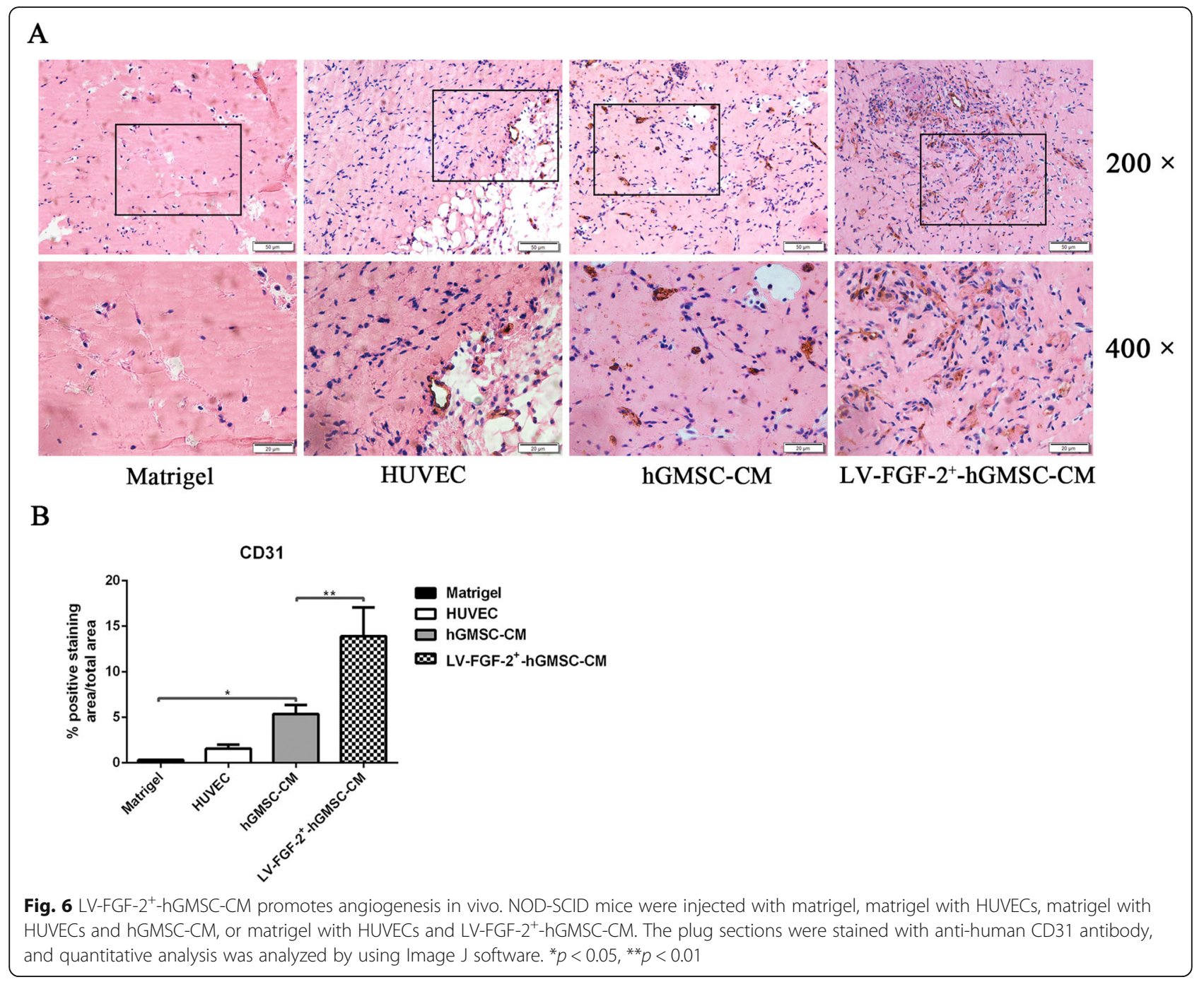

such as VEGF, FGF-2, and TGF- $\beta$ in hGMSCs. LV-FGF$2^{+}$-hGMSC-CM had a stronger pro-angiogenesis and migration promotion effect on HUVECs than LV-vector ${ }^{+}-$ hGMSC-CM.

Growth factor VEGF has been a hot spot for studying angiogenesis, and its role in angiogenesis and tissue repair or regeneration has attracted wide attention [23, 24, 33-36]. It can enhance both intra-articular ossification and intramembranous bone formation [34, 37], meanwhile can recruit stem cells to damaged or diseased bone tissue $[33,36]$. However, studies have also shown that the use of VEGF alone does not promote the healing of bone damage and the combination with other growth factors has a stronger and more comprehensive role in promoting tissue healing [35]. TGF- $\beta$ plays an important role in the physiological and pathological processes associated with tissue remodeling. In terms of angiogenesis, TGF- $\beta$ upregulates the production of TSP-4, a proangiogenic extracellular matrix protein in cultured endothelial cells (EC) [38], thereby promoting angiogenesis. It also augments ECM protein expression via the SMAD signaling pathway to remodel ECM to promote blood vessel production [39]. Given the importance of VEGF and TGF- $\beta$ in angiogenesis, elevating their concentration should promote angiogenesis potentials. In this study, FGF-2 gene transfection increased the secretion of VEGF and TGF- $\beta$ in hGMSCs. Importantly, FGF- 2 itself is an important inducer for angiogenesis. All these suggest that FGF-2-overexpressed hGMSCs may be a perspective medication tool for angiogenesis.

Successful bone defect healing requires simultaneous regeneration of both the mineralized tissue and vasculature to obtain the highly vascularized bone. During the vasculature formation process, a variety of mediators of angiogenesis induce migration and differentiation of endothelial progenitor cells (EPCs) to increase blood vessel density [40]. In this study, the effect of the CM obtained from FGF-2 overexpressed hGMSCs on HUVEC angiogenic ability was evaluated in vitro and in vivo. The results confirmed that hGMSC-CM 
enhanced gene and protein expressions of angiogenesisrelated factors, tube-forming ability, and cell migration ability in HUVECs while LV-FGF- $2^{+}$-hGMSC-CM had stronger efficacy compared with hGMSC-CM.

CD31, also known as platelet endothelial cell adhesionmolecule1 (PEMCAM1), is a differentiation marker of blood vessel EPCs [41]. It is one of the most famous immunohistochemical markers among vascular tumors, and its expression level is often used to reflect blood vessel density [42, 43]. In this study, we observed the expression level of CD31 in matrigel plug sections by immunohistochemistry. It was found that although hGMSC-CM promoted some blood vessel formation, the $\mathrm{CM}$ derived from FGF-2 gene-modified hGMSCs promoted more blood vessel formation.

Constitutive overexpression of FGF-2 in MSCs has a safety backlash. Just as pointed out by Fierro et al. [44], extensive safety and efficacy evaluation must be done before this type of cell/gene therapy could ever be considered. However, our proposed strategy is to promote the concentration of FGF2 and other proangiogenic factors in the $\mathrm{CM}$, while the application of the $\mathrm{CM}$ is controllable. Recombinant human fibroblast growth factor (rhFGF)-2 has been used clinically in periodontal regeneration therapy [45], whereas FGF-2modified MSC-derived CM provides some advantage over exogenous supplement of FGF-2 into the CM in that FGF-2-modified GMSCs not only produce and release more FGF-2, but also more VEGF-A, and TGF- $\beta$ vs primary GMSCs. Briefly, an optimized conditioned medium by overexpressing the FGF-2 gene potentiates the angiogenic ability of hGMSCs.

\section{Conclusion}

In summary, the present investigation reveals that $\mathrm{CM}$ from FGF-2-modified hGMSCs contains more VEGF$\mathrm{A}$, FGF-2, and TGF- $\beta$ and has a stronger proangiogenic and migration promotion effect on HUVECs than on hGMSC-CM in vitro and in vivo. Nevertheless, many further tasks need to be conducted to evaluate the advantage of this strategy. For example, the deeper in vivo experiment is required to observe whether FGF2-modified hGMSC-CM promotes a simultaneous regeneration of both the mineralized tissue and vasculature to obtain the highly vascularized bone.

\section{Supplementary information}

Supplementary information accompanies this paper at https://doi.org/10. 1186/s13287-020-1584-3.

Additional file 1. Plasmid map. The map of plasmid showed that the lentivirus vector pHBLV-CMV-MCS-3FLAG-EF1-ZsGreen-T2A-PURO-FGF-2 was successfully constructed.

\section{Abbreviations}

BMMSCs: Bone marrow mesenchymal stem cells; CKO: Conditional knockout: CM: Conditioned medium; FGF-2: Fibroblast growth factor-2;

hGMSCs: Human gingival MSCs; HUVECs: Human umbilical vein endothelial cells; LV-FGF-2+-hGMSCs: FGF-2 gene-modified hGMSCs were constructed by lentiviral transfection technology; MSC-CM: Conditioned medium derived from mesenchymal stem cells; MSCs: Mesenchymal stem cells; PDLSCs: Stem cells from periodontal ligaments; PLGF: Placenta growth factor; SCF: Stem cell factor; TGF- $\beta$ : Transforming growth factor $\beta$; VEGF-A: Vascular endothelial growth factor A; VEGFR2: Vascular endothelial growth factor receptor 2

\section{Acknowledgements \\ Not applicable.}

\section{Authors' contributions}

PY and QS conceived the project. SJ was responsible for the experimental design and application. QS, CY, and HJ supervised the study. SJ and PY wrote and revised the manuscript. $L L, Y Z, S L$, and $L Z$ analyzed the data. All authors read and approved the final manuscript.

\section{Funding}

This study was supported by the National Natural Science Foundation of China (grant no. 81771076).

\section{Availability of data and materials}

The datasets used and/or analyzed during the current study are included in this published article or available from the corresponding author on reasonable request.

\section{Ethics approval and consent to participate}

This study was approved by the Ethics Committee of the Stomatological Hospital of Shandong University (No. 201712).

\section{Consent for publication}

Not applicable.

\section{Competing interests}

The authors declare that they have no competing interests.

\section{Author details}

${ }^{1}$ Department of Periodontology, School and Hospital of Stomatology, Shandong University, No.44-1 Wenhua Road West, Jinan 250012, Shandong, China. ${ }^{2}$ Shandong Provincial Key Laboratory of Oral Tissue Regeneration, Jinan, Shandong, China. ${ }^{3}$ Shandong Engineering Laboratory for Dental Materials and Oral Tissue Regeneration, Jinan, Shandong, China. ${ }^{4}$ Department of Stomatology, Qilu Hospital of Shandong University, Jinan, Shandong, China.

Received: 15 November 2019 Revised: 19 January 2020 Accepted: 4 February 2020 Published online: 18 February 2020

\section{References}

1. Zhou Q, et al. The promotional effect of mesenchymal stem cell homing on bone tissue regeneration. Curr Stem Cell Res Ther. 2017;12(5):365-76.

2. Almubarak $S$, et al. Tissue engineering strategies for promoting vascularized bone regeneration. Bone. 2016;83:197-209.

3. Zhang $L$, et al. CXCL12 overexpression promotes the angiogenesis potential of periodontal ligament stem cells. Sci Rep. 2017;7(1):10286.

4. Ahmad O, et al. Bone regenerative medicine: classic options, novel strategies, and future directions. J Orthop Surg Res. 2014;9(1):18-799X-9-18.

5. Gaihre B, et al. Reconstruction of craniomaxillofacial bone defects using tissue-engineering strategies with injectable and non-injectable scaffolds. J Funct Biomater. 2017:8(4):49.

6. Sheikh Z, et al. Natural graft tissues and synthetic biomaterials for periodontal and alveolar bone reconstructive applications: a review. Biomater Res. 2017;21:9.

7. Hass R, et al. Different populations and sources of human mesenchymal stem cells (MSC): a comparison of adult and neonatal tissue-derived MSC. Cell Commun Signal. 2011;9:12. 
8. Seo B-M, et al. Investigation of multipotent postnatal stem cells from human periodontal ligament. Lancet. 2004;364(9429):149-55.

9. Wang ZS, et al. The use of platelet-rich fibrin combined with periodontal ligament and jaw bone mesenchymal stem cell sheets for periodontal tissue engineering. Sci Rep. 2016;6:28126.

10. Gronthos S, et al. Postnatal human dental pulp stem cells (DPSCs) in vitro and in vivo. Proc Natl Acad Sci U S A. 2000 Dec 5;97(25):13625-30.

11. Liu $Y$, et al. PD-1 is required to maintain stem cell properties in human dental pulp stem cells. Cell Death Differ. 2018;25(7):1350-60.

12. Xu Q-C, et al. Systemically transplanted human gingiva-derived mesenchymal stem cells contributing to bone tissue regeneration. Int J Clin Exp Pathol. 2014;7(8):4922-9.

13. Zhang $\mathrm{Q}$, et al. Mesenchymal stem cells derived from human gingiva are capable of immunomodulatory functions and ameliorate inflammationrelated tissue destruction in experimental colitis. J Immunol. 2009;183(12): 7787-98.

14. Sui BD, et al. Stem cell-based bone regeneration in diseased microenvironments: challenges and solutions. Biomaterials. 2019;196:18-30

15. Marx J. Cancer research: mutant stem cells may seed cancer. Science. 2003; 301(5638):1308-10.

16. Menasche P. Stem cells for clinical use in cardiovascular medicine - current limitations and future perspectives. Thromb Haemost. 2005;94(4):697-701.

17. Yu M, et al. Osteoblast progenitors enhance osteogenic differentiation of periodontal ligament stem cells. J Periodontol. 2017;88(10):e159-68.

18. Tasso R, et al. Recruitment of a host's osteoprogenitor cells using exogenous mesenchymal stem cells seeded on porous ceramic. Tissue Eng A. 2009:15(8):2203-12.

19. Chen J, et al. Mesenchymal stem cell conditioned medium promotes proliferation and migration of alveolar epithelial cells under septic conditions in vitro via the JNK-P38 signaling pathway. Cell Physiol Biochem. 2015;37(5):1830-46.

20. Chen $\mathrm{H}$, et al. Pre-activation of mesenchymal stem cells with TNF-alpha, IL1 beta and nitric oxide enhances its paracrine effects on radiation-induced intestinal injury. Sci Rep. 2015;5:8718.

21. Duijvestein $\mathbf{M}$, et al. Pretreatment with interferon-gamma enhances the therapeutic activity of mesenchymal stromal cells in animal models of colitis. Stem Cells. 2011;29(10):1549-58.

22. Liu JJ, et al. Noninvasive ventilation in patients with hematologic malignancy: a retrospective study. J Intensive Care Med. 2019;34(3):197-203.

23. Zhang $Y$, et al. Protein Phosphotyrosine phosphatase 1B (PTP1B) in Calpaindependent feedback regulation of vascular endothelial growth factor receptor (VEGFR2) in endothelial cells: implications in VEGF-dependent angiogenesis and diabetic wound healing. J Biol Chem. 2017;292(2):407-16.

24. Zelzer E. VEGFA is necessary for chondrocyte survival during bone development. Development. 2004;131(9):2161-71.

25. Suuronen EJ, et al. Comparative effects of mesenchymal progenitor cells, endothelial progenitor cells, or their combination on myocardial infarct regeneration and cardiac function. J Thorac Cardiovasc Surg. 2007;134(5): 1249-58.

26. Piard C, et al. 3D printed HUVECs/MSCs cocultures impact cellular interactions and angiogenesis depending on cell-cell distance. Biomaterials. 2019;222:119423.

27. Wu J, et al. Comparative study of heparin-poloxamer hydrogel modified bFGF and aFGF for in vivo wound healing efficiency. ACS Appl Mater Interfaces. 2016;8(29):18710-21.

28. Yafai $Y$, et al. Basic fibroblast growth factor contributes to a shift in the angioregulatory activity of retinal glial (Muller) cells. PLoS One. 2013;8(7): e68773

29. Hollborn $\mathrm{M}$, et al. Characterization of the basic fibroblast growth factorevoked proliferation of the human Muller cell line, MIO-M1. Graefes Arch Clin Exp Ophthalmol. 2004:242(5):414-22.

30. Tasso R, et al. The role of bFGF on the ability of MSC to activate endogenous regenerative mechanisms in an ectopic bone formation model. Biomaterials. 2012;33(7):2086-96.

31. Du M, et al. Acellular dermal matrix loading with bFGF achieves similar acceleration of bone regeneration to BMP-2 via differential effects on recruitment, proliferation and sustained osteodifferentiation of mesenchymal stem cells. Mater Sci Eng C Mater Biol Appl. 2017;70(Pt 1):62-70.

32. Zhang $C$, et al. The biological behavior optimization of human periodontal ligament stem cells via preconditioning by the combined application of fibroblast growth factor-2 and A83-01 in in vitro culture expansion. J Transl Med. 2019;17(1):66.

33. Beamer B, et al. Vascular endothelial growth factor: an essential component of angiogenesis and fracture healing. HSS J. 2010;6(1):85-94.

34. Geiger F, et al. VEGF producing bone marrow stromal cells (BMSC) enhance vascularization and resorption of a natural coral bone substitute. Bone. 2007:41(4):516-22

35. Kasten $\mathrm{P}$, et al. Comparison of platelet-rich plasma and VEGF-transfected mesenchymal stem cells on vascularization and bone formation in a criticalsize bone defect. Cells Tissues Organs. 2012;196(6):523-33.

36. Keramaris NC, et al. Fracture vascularity and bone healing: a systematic review of the role of VEGF. Injury. 2008;39:S45-57.

37. Li R, et al. Effect of cell-based VEGF gene therapy on healing of a segmental bone defect. J Orthop Res. 2009;27(1):8-14.

38. Muppala S, et al. Thrombospondin-4 mediates TGF-beta-induced angiogenesis. Oncogene. 2017;36(36):5189-98.

39. Goumans MJ, et al. TGF-beta signaling in control of cardiovascular function. Cold Spring Harb Perspect Biol. 2018;10:a022210.

40. Wagner DR, et al. Dysfunctional stem and progenitor cells impair fracture healing with age. World J Stem Cells. 2019;11(6):281-96.

41. Cui $X$, et al. Shear stress augments the endothelial cell differentiation marker expression in late EPCs by upregulating integrins. Biochem Biophys Res Commun. 2012;425(2):419-25.

42. Razavi $\mathrm{S}$, et al. Comparative study of correlation between angiogenesis markers (CD31) and Ki67 marker with behavior of aggressive and nonaggressive central giant cell granuloma with immunohistochemistry technique. Asian Pac J Cancer Prev. 2018;19(8):2279-83.

43. Yao $Y$, et al. Prognostic significance of microvessel density determined by an anti-CD105_endoglin monoclonal antibody in astrocytic tumors comparison with an anti-CD31 monoclonal antibody. Neuropathology. 2005 Sep.;25(3):201-6.

44. Fierro FA, et al. Effects on proliferation and differentiation of multipotent bone marrow stromal cells engineered to express growth factors for combined cell and gene therapy. Stem Cells. 2011;29(11):1727-37.

45. Saito A, Bizenjima T, Takeuchi T, et al. Treatment of intrabony periodontal defects using rhFGF-2 in combination with deproteinized bovine bone mineral or rhFGF-2 alone: a 6-month randomized controlled trial. J Clin Periodontol. 2019;46(3):332-41.

\section{Publisher's Note}

Springer Nature remains neutral with regard to jurisdictional claims in published maps and institutional affiliations.

\section{Ready to submit your research? Choose BMC and benefit from:}

- fast, convenient online submission

- thorough peer review by experienced researchers in your field

- rapid publication on acceptance

- support for research data, including large and complex data types

- gold Open Access which fosters wider collaboration and increased citations

- maximum visibility for your research: over $100 \mathrm{M}$ website views per year

At BMC, research is always in progress.

Learn more biomedcentral.com/submission 